\title{
Pulverização de glyphosate utilizando solução de uréia + sulfato de amônio ${ }^{1}$
}

\author{
Glyphosate application using solution of urea + ammonium sulfate
}

Saul Jorge Pinto Carvalho ${ }^{2}$, Virginia Damin ${ }^{3}$, Ana Carolina Ribeiro Dias ${ }^{4}$, Heryaldo Tarozzo

Filho $^{5}$, Pedro Jacob Christoffoleti ${ }^{6}$

Resumo - Diversos experimentos têm sido realizados com intuito de elevar a eficácia do herbicida glyphosate na dessecação de plantas daninhas, incluindo-se o uso de sulfato de amônio ou ureia como adjuvantes. Em termos fisiológicos, existem explicações para o efeito complementar destes produtos quando adicionados concomitantemente à calda herbicida. Desta forma, três experimentos foram desenvolvidos em campo com o objetivo de avaliar a eficácia do herbicida glyphosate para dessecação de espécies de plantas daninhas utilizando solução de fertilizantes nitrogenados como veículos de pulverização. Foram avaliados seis tratamentos, sendo estes: aplicação de glyphosate (540 g e.a. ha $\left.{ }^{-1}\right)$ utilizando água, solução de sulfato de amônio - SA $\left(15 \mathrm{~g} \mathrm{~L}^{-1}\right)$, solução de uréia - U $\left(5 \mathrm{~g} \mathrm{~L}^{-1}\right)$, solução de $\mathrm{U}+\mathrm{SA}\left(2,5+7,5 \mathrm{~g} \mathrm{~L}^{-1}\right)$ como veículos de pulverização, glyphosate a $1080 \mathrm{~g}$ e.a. ha ${ }^{-1}$ aplicado com água, e testemunha sem aplicação. Avaliou-se a eficácia dos tratamentos sobre comunidade mista de plantas daninhas, Brachiaria decumbens e Sorghum halepense. O uso de solução de fertilizantes nitrogenados como veículo para pulverização do glyphosate não elevou o controle de $B$. decumbens. A menor dose do herbicida glyphosate teve maior eficácia sobre a comunidade mista de plantas daninhas e sobre $S$. halepense devido à pulverização com solução de $\mathrm{SA}\left(15 \mathrm{~g} \mathrm{~L}^{-1}\right)$ ou de U+SA $(2,5+7,5 \mathrm{~g}$ $\left.\mathrm{L}^{-1}\right)$. Os resultados positivos obtidos pela aplicação de glyphosate com fertilizantes nitrogenados também foram observados para a redução de massa fresca da comunidade mista e para massa fresca e seca de $S$. halepense.

Palavras-chave: eficácia, fertilizantes, Sorghum halepense, Brachiaria decumbens.

\begin{abstract}
Several trials have been carried out for increasing glyphosate efficacy on weed burndown, including the addiction of ammonium sulfate and urea as adjuvants. On a physiological approach, there are explanations to complementary effect of these fertilizers when concomitantly added to herbicide spray solution. Therefore, three experiments were developed in field conditions with the objective of evaluating glyphosate efficacy for burning down different weed species by using solution of nitrogen fertilizers as spraying vehicles. Six treatments were evaluated, as follows: glyphosate (540 g a.e. ha $\left.^{-1}\right)$ application using water, solution of ammonium sulfate - AMS $\left(15 \mathrm{~g} \mathrm{~L}^{-1}\right)$, solution of urea - U $\left(5 \mathrm{~g} \mathrm{~L}^{-1}\right)$, solution of U+AMS $\left(2.5+7.5 \mathrm{~g} \mathrm{~L}^{-1}\right)$ as

\footnotetext{
${ }^{1}$ Recebido para publicação em 12/12/2011 e aceito em 11/02/2012.

${ }^{2}$ Professor do Instituto Federal do Sul de Minas Gerais, Campus Machado. E-mail: sjpcarvalho@yahoo.com.br;

${ }^{3}$ Professora da Universidade Federal de Goiás. E-mail: virginiadamin@ gmail.com;

${ }^{4}$ Doutoranda em Fitotecnia pela Esalq/USP. E-mail: anacarolina.r.dias@gmail.com;

${ }^{5}$ Engenheiro Agrônomo, Syngenta Proteção de Cultivos. E-mail: heryaldo@yahoo.com;

${ }^{6}$ Professor Associado, Esalq/USP. E-mail: pjchrist@esalq.usp.br
} 
Carvalho et al.

spraying vehicles, glyphosate at $1080 \mathrm{~g}$ a.e. ha $^{-1}$ sprayed with water, and check without application. Treatment's efficacy was evaluated on mixed community of weeds, Brachiaria decumbens and Sorghum halepense. The use of nitrogen fertilizer solutions as glyphosate spraying vehicles did not increase $B$. decumbens control. The lower rate of glyphosate had higher efficacy over mixed weed community and over $S$. halepense due to the use of ammonium sulfate $\left(15 \mathrm{~g} \mathrm{~L}^{-1}\right)$ or $\mathrm{U}+\mathrm{AMS}\left(2,5+7,5 \mathrm{~g} \mathrm{~L}^{-1}\right)$ solution as spraying vehicles. The positive results reached after glyphosate application with nitrogen fertilizers were also observed on the reduction of the mixed weed community fresh mass and for S. halepense fresh and dry mass.

Key words: efficacy, fertilizer, Sorghum halepense, Brachiaria decumbens.

\section{Introdução}

Dentre os fatores bióticos presentes em um agroecossistema, as plantas daninhas são um dos principais componentes que interferem negativamente nas culturas. Os efeitos negativos causados pelas plantas daninhas se manifestam sobre a quantidade e a qualidade do produto colhido, consequência da competição pelos recursos de crescimento oferecidos pelo ambiente, da alelopatia ou por serem hospedeiras de pragas e doenças. Lorenzi (2006) estima que, no Brasil, as perdas ocasionadas às culturas agrícolas pela interferência das plantas daninhas sejam da ordem de 20 a $30 \%$.

Atualmente, o principal método de controle de plantas daninhas é o químico, por meio da aplicação de herbicidas em pré ou pósemergência das plantas daninhas e/ou das culturas. Neste sentido, o advento das culturas geneticamente modificadas para tolerância a herbicidas (transgênicas) e os sistemas de produção do tipo conservacionistas (semeadura direta) contribuíram para a crescente adoção do herbicida glyphosate (Young et al., 2003; Christoffoleti et al., 2008; Carvalho et al., 2008).

Trata-se de um herbicida não-seletivo, de ação sistêmica, usado no controle de plantas daninhas anuais e perenes e na dessecação de culturas de cobertura (Rodrigues \& Almeida, 2005; Timossi et al., 2006). A molécula inibe a EPSPs (enol piruvil shiquimato fosfato sintase), enzima que participa da rota de síntese dos aminoácidos aromáticos fenilalanina, tirosina e triptofano (Geiger \& Fuchs, 2002). Sua absorção é um processo bifásico, que envolve rápida penetração pela cutícula, seguida de absorção simplástica lenta, dependendo da idade da planta, ambiente, surfactantes e concentração do herbicida (Monquero et al., 2004).

Visando facilitar a penetração foliar do herbicida, bem como a absorção celular, diversas substâncias têm sido adicionadas à calda de pulverização, com destaque para a uréia e o sulfato de amônio (Carvalho et al., 2009a,b; 2010). O principal mecanismo de incremento de controle relatado para a uréia diz respeito à contribuição na penetração cuticular, devido à difusão facilitada desta molécula, e ao rompimento de ligações éster, éter e di-éter da cutina (Yamada et al., 1965; Witte et al., 2002). Comumente, a maior eficácia do herbicida glyphosate em presença do sulfato de amônio é atribuída à atividade do sulfato $\left(\mathrm{SO}_{4}{ }^{2-}\right.$ ) como antagonista de cátions presentes na água, sobretudo $\mathrm{Ca}^{2+}$ e $\mathrm{Mg}^{2+}$ (Nalewaja \& Matysiak, 1991; Salas-Pino, 1996; Gauvrit, 2003). Também há relatos sobre a formação de sais glyphosate-amônio que possuem maior facilidade de absorção (Nalewaja et al., 1992; Thelen et al., 1995); além da acidificação do apoplasto foliar, que facilita a absorção celular de herbicidas ácido fracos (Gronwald et al., 1993; Ruiter \& Meinen, 1996; Young et al., 
Carvalho et al.

2003). Ainda, MacIsaac et al. (1991) comentam que a adição de sulfato de amônio à calda promove alteração na morfologia das gotas, atrasando ou prevenindo a cristalização do glyphosate na superfície foliar.

Em resumo, a uréia pode contribuir para a penetração cuticular do glyphosate, enquanto o sulfato de amônio é condicionador da água, além de promover a acidificação do apoplasto (Carvalho et al., 2010). Portanto, há indícios de efeito complementar destes fertilizantes nitrogenados, contudo não foram encontrados trabalhos que avaliaram a adição concomitante dos mesmos à calda herbicida em condição de campo. Neste sentido, este trabalho foi desenvolvido em campo com o objetivo de avaliar a eficácia do herbicida glyphosate para dessecação de diferentes espécies de plantas daninhas utilizando solução de sulfato de amônio (SA), uréia (U) e U+SA como veículos de pulverização.

\section{Material e métodos}

O trabalho foi desenvolvido no município de Piracicaba/SP (22 $2^{\circ} 42^{\prime} \quad 30^{\prime \prime}$ Latitude Sul, $47^{\circ} 38^{\prime} 00^{\prime \prime}$ Longitude Oeste e 546 $\mathrm{m}$ de altitude), no período compreendido entre
Janeiro e Março de 2009. Foram instalados três experimentos semelhantes em campo, em áreas distintas quanto à infestação de plantas daninhas, todas pertencentes ao Departamento de Produção Vegetal da Escola Superior de Agricultura "Luiz de Queiroz" (Esalq/USP).

Nos experimentos, utilizou-se o delineamento experimental de blocos ao acaso com seis tratamentos e seis repetições. Nas três áreas foram aplicados os mesmos tratamentos herbicidas, que estão detalhadamente descritos na Tabela 1; fundamentados na aplicação de glyphosate (Roundup Original ${ }^{\circledR}$ ), em dose de 540 g e.a. ha ${ }^{-1}$, utilizando os seguintes veículos de pulverização: água, solução de sulfato de amônio - SA (15 $\left.\mathrm{g} \mathrm{L}^{-1}\right)$, solução de uréia - U (5 $\left.\mathrm{g} \mathrm{L}^{-1}\right)$, solução de $\mathrm{U}+\mathrm{SA}\left(2,5+7,5 \mathrm{~g} \mathrm{~L}^{-1}\right)$, glyphosate a $1080 \mathrm{~g}$ e.a. ha $^{-1}$ aplicado com água; além de testemunha sem aplicação. Os fertilizantes nitrogenados utilizados foram provenientes de fontes convencionalmente utilizadas nas adubações agrícolas, com concentrações de 21 e $45 \%$ de nitrogênio no sulfato de amônio e uréia, respectivamente. As doses utilizadas foram obtidas em experimentos desenvolvidos por Carvalho et al. (2008; 2010).

Tabela 1. Tratamentos herbicidas aplicados em campo.

\begin{tabular}{|c|c|c|c|c|c|}
\hline \multirow{3}{*}{$\mathrm{N}^{\circ}$} & \multicolumn{2}{|c|}{ Herbicida } & \multicolumn{3}{|c|}{ Fertilizante Nitrogenado } \\
\hline & \multirow{2}{*}{ Condição } & \multirow{2}{*}{$\begin{array}{c}\text { Dose } \\
\left(\mathrm{g} \mathrm{e}^{\mathrm{a} .} \mathrm{ha}^{-1}\right)\end{array}$} & \multirow{2}{*}{ Tipo } & \multicolumn{2}{|c|}{ Dose $^{(2)}$} \\
\hline & & & & $\left(\mathrm{g} \mathrm{L}^{-1}\right)$ & $\left(\mathrm{kg} \mathrm{ha}^{-1}\right)$ \\
\hline 1 & Testemunha & a aplicação & Ausente & 0,0 & 0,0 \\
\hline 2 & Glyphosate $^{(1)}$ & 540 & Ausente & 0,0 & 0,0 \\
\hline 3 & Glyphosate & 540 & Sulfato de Amônio & 15,0 & 4,5 \\
\hline 4 & Glyphosate & 540 & Uréia & 5,0 & 1,5 \\
\hline 5 & Glyphosate & 540 & Uréia + Sulfato de Amônio & $2,5+7,5$ & $0,75+2,25$ \\
\hline 6 & Glyphosate & 1080 & Ausente & 0,0 & 0,0 \\
\hline
\end{tabular}

${ }^{(1)}$ Roundup Original ${ }^{\circledR}, 360 \mathrm{~g} \mathrm{~L}^{-1}$ de equivalente ácido; ${ }^{(2)}$ Volume de calda proporcional a $300 \mathrm{~L} \mathrm{ha}^{-1}$.

O primeiro experimento foi instalado em área cujo solo foi classificado como NITOSSOLO VERMELHO eutrófico (EMBRAPA, 2006) de textura argilosa, $\mathrm{pH} 5,4$, com 2,5\% de matéria orgânica e $69 \%$ de saturação por bases. Constatou-se intensa infestação por plantas daninhas na área (média de 45 plantas $\mathrm{m}^{-2}$ ), com presença das seguintes espécies, todas em florescimento: $60 \%$ de apaga-fogo (Alternanthera tenella), $15 \%$ de 
Carvalho et al.

losna-branca (Parthenium hysterophorus), 10\% de caruru-de-mancha (Amaranthus viridis), $10 \%$ de corda-de-viola (Ipomoea nil) e $5 \%$ de outras espécies. As parcelas foram delimitadas em 2,5 x 3,0 m, considerando-se $6 \mathrm{~m}^{2}$ de área útil.

O segundo experimento foi desenvolvido em área de aterro com cerca de 2,5 $\mathrm{m}$ de altura, relevo plano, solo de textura média-argilosa, $\mathrm{pH} 4,5$, com $2,1 \%$ de matéria orgânica e 39\% de saturação por bases. Constatou-se densa infestação de capimbraquiária (Brachiaria decumbens), considerada como espécie dominante (96\%), cuja densidade foi estimada em 180 perfilhos $\mathrm{m}^{-2}$. As parcelas foram delimitadas em $3 \times 4 \mathrm{~m}$, considerando-se $7,5 \mathrm{~m}^{2}$ de área útil. No momento da aplicação dos tratamentos, registrou-se estádio fenológico de florescimento/frutificação para o capimbraquiária.

O terceiro experimento foi desenvolvido em área cujo solo também foi classificado como Nitossolo Vermelho eutrófico (EMBRAPA, 2006) de textura argilosa, relevo com suave declive, $\mathrm{pH} 5,4$, com 3,2\% de matéria orgânica e $75 \%$ de saturação por bases. Constatou-se densa infestação de capim-massambará (Sorghum halepense), considerada como espécie dominante (94\%), cuja densidade foi estimada em 90 perfilhos $\mathrm{m}^{-2}$. As parcelas foram delimitadas em $3 \times 4 \mathrm{~m}$, considerando-se 7,5 $\mathrm{m}^{2}$ de área útil. No momento da aplicação dos tratamentos, registrou-se estádio fenológico de pleno perfilhamento / pré-florescimento para o capim-massambará.

Em todas as aplicações, utilizou-se pulverizador costal pressurizado por $\mathrm{CO}_{2}$, acoplado a uma barra de pulverização com $2 \mathrm{~m}$ de largura, com quatro pontas do tipo jato plano, modelo XR 110.02, espaçadas em 0,50 $\mathrm{m}$, calibrado para volume de calda proporcional a $300 \mathrm{~L} \mathrm{ha}^{-1}$. O maior volume de calda foi adotado visando potencializar os efeitos oriundos da utilização de soluções de fertilizantes nitrogenados como veículo de pulverização, uma vez que a eficácia do glyphosate tende a ser reduzida nestas condições (O'Sullivan et al., 1981; Salas-Pino, 1996; Ramsdale et al., 2003).

$\mathrm{Na}$ primeira área, com comunidade mista de plantas daninhas, a pulverização foi realizada dia 07/01/2009, das 10:40 às 11:00h, quando registraram-se ventos de $2,4 \mathrm{~km} \mathrm{~h}^{-1}$, $78,0 \%$ de umidade relativa do ar (UR \%), temperatura de $25,6^{\circ} \mathrm{C}$ e céu sem nuvens. Na segunda área, com infestação de $B$. decumbens, a pulverização foi realizada dia 20/02/2009, das 08:55 às 09:25h, quando registraram-se ventos de $1,8 \mathrm{~km} \mathrm{~h}^{-1}, 88,2 \%$ de $\mathrm{UR}(\%)$, temperatura de $24,8^{\circ} \mathrm{C}$ e ausência de nuvens. $\mathrm{Na}$ terceira área, com infestação de $S$. halepense, a pulverização foi realizada dia 03/03/2009, das 09:35 às 10:15h, quando registraram-se ventos de $1,6 \mathrm{~km} \mathrm{~h}^{-1}, 91,5 \%$ de $\mathrm{UR}(\%)$, temperatura de $27,5^{\circ} \mathrm{C}$ e céu parcialmente encoberto (50\%). Adicionalmente, dados de precipitação e temperatura média registrados durante $o$ período de desenvolvimento dos experimentos em campo estão apresentados na Figura 1. Em todos os casos, a primeira chuva foi registrada em período superior a 48 horas após aplicação.

A água utilizada nas aplicações passou por tratamento comum para distribuição pública, realizado na Esalq/USP e o resultado de sua análise foi $\left(\mathrm{mg} \mathrm{L}^{-1}\right)$ : cloreto, 43,6; nitrato, 4,0; sulfato, 33,0; fósforo, 0,02; sódio, 66,0; potássio, 5,6; cálcio, 31,8; magnésio, 2,8; ferro, 0,0; cobre, 0,0; manganês, 0,01 zinco, 0,04 alcalinidade $\left(2 \mathrm{CO}_{3}{ }^{2-}+\mathrm{HCO}_{3}{ }^{-}\right), 49,6$; nitrogênio amoniacal, 0,2; sedimentos em suspensão, 4,3; gás carbônico, 2,5; acidez $\left(\mathrm{CaCO}_{3}\right), 5,0$; e dureza total $\left(\mathrm{CaCO}_{3}\right)$, 91,0. Os demais parâmetros apresentaram os seguintes valores: $\mathrm{pH}, 7,5$; cor aparente, 0 PtCo; turbidez, 0 FTU; e condutividade elétrica (CE), $0,33 \mathrm{mS} \mathrm{cm} \mathrm{cm}^{-1}$ A dureza total foi calculada com base no equivalente de 
Carvalho et al.

carbonato de cálcio $\left(\mathrm{CaCO}_{3}\right)$, segundo Franson $\left.{ }^{1}\right]$. (1995): 2,497 [Ca, $\left.\mathrm{mg} \mathrm{L}^{-1}\right]+4,118\left[\mathrm{Mg}, \mathrm{mg} \mathrm{L}^{-}\right.$

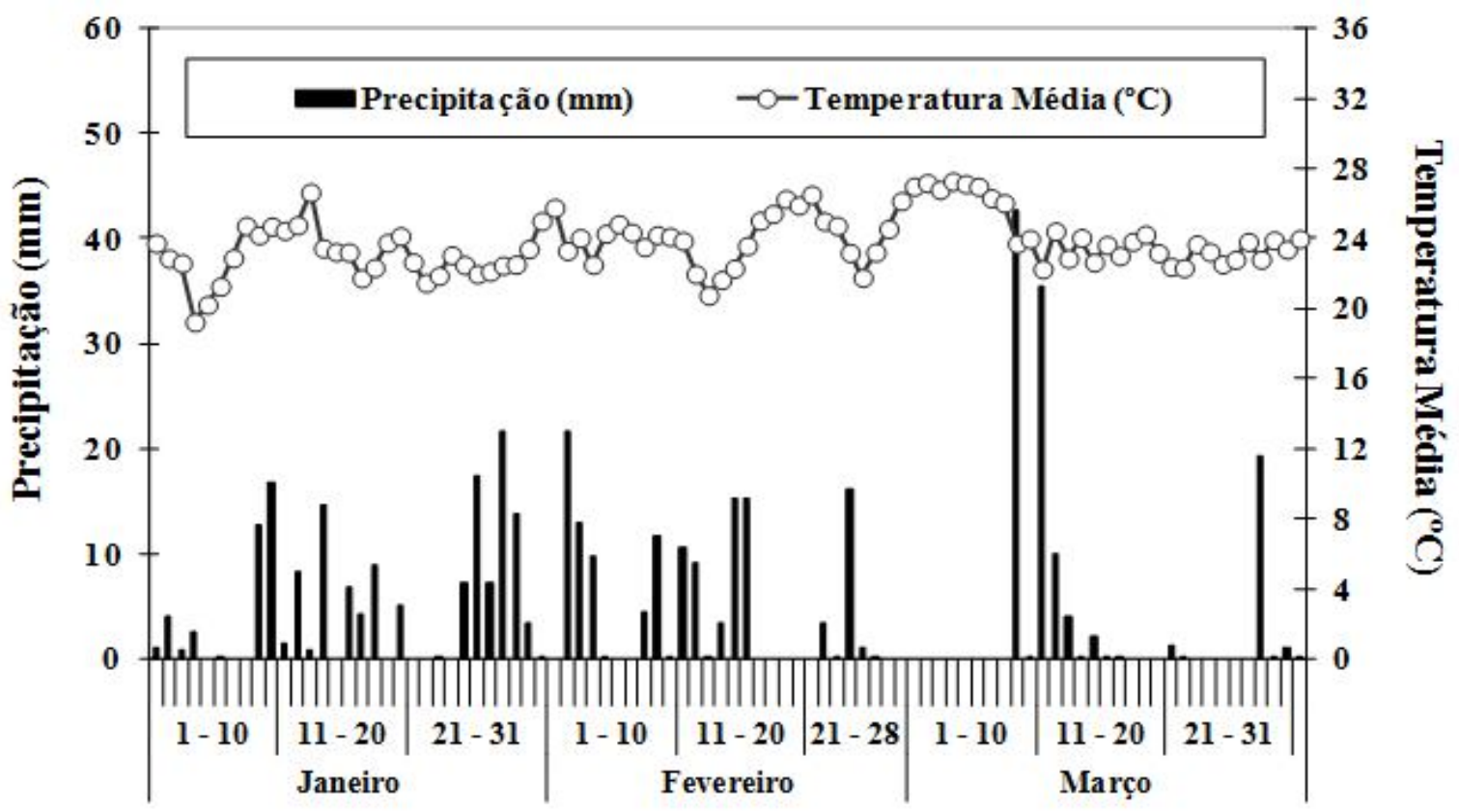

\section{Meses / Dias (2009)}

Figura 1. Temperatura média $\left({ }^{\circ} \mathrm{C}\right)$ e precipitações $(\mathrm{mm})$ diárias observadas durante o período de condução dos experimentos em campo. Piracicaba, 2009

As variáveis avaliadas foram controle percentual aos 14, 21 e 28 dias após a aplicação (DAA) e massa fresca e seca residual aos 28 DAA. Para as avaliações de controle, utilizouse escala com variação entre 0 e $100 \%$, em que zero representou a ausência de sintomas e 100 a morte de todas as plantas (ALAM, 1974). Para massa fresca e seca, em campo, um gabarito de ferro de $0,5 \times 0,5 \mathrm{~m}$ foi lançado uma vez em área representativa de cada parcela. Todo o material vegetal presente na área do quadrado foi cortado rente à superfície do solo e secado em estufa $\left(70^{\circ} \mathrm{C}\right)$ por $72 \mathrm{~h}$. Posteriormente, os dados de massa foram convertidos para $\mathrm{g} \mathrm{m}^{-2}$.

Os dados obtidos em campo foram submetidos à análise por grupo de experimentos, com aplicação do teste $\mathrm{F}$ na análise da variância. Quando os efeitos dos tratamentos foram significativos, aplicou-se teste de Scott-Knott para agrupamentos das médias (Scott \& Knott, 1974), com 5\% de significância.

\section{Resultados e discussão}

$\mathrm{Na}$ análise por grupos de experimentos, é pressuposto que a variância dos erros experimentais dos diferentes experimentos seja homogênea (Storck et al., 2006). Para avaliar a homocedasticidade dos experimentos, utilizouse teste de F máximo, que indicou diferenças nos quadrados médios dos resíduos, inviabilizando a análise conjunta das áreas. Assim, cada experimento foi analisado individualmente e os resultados de controle percentual e massa residual das plantas daninhas estão apresentados nas Tabelas 2 e 3, respectivamente.

Com relação ao controle percentual das 
Carvalho et al.

diferentes espécies de plantas daninhas, observou-se constância de resultados nas diferentes avaliações (Tabela 2). Quando se estudou a comunidade mista (Área 1), todos os tratamentos herbicidas proporcionaram controles superiores a $80 \%$ nas três datas de avaliação. Não foi observado incremento de controle para aplicação de glyphosate com solução de uréia (U), que teve eficácia igual à menor dose de glyphosate $\left(540 \mathrm{~g} \mathrm{ha}^{-1}\right)$ aplicada com água. Mesmo utilizando-se água com baixa concentração de sais, foi constatado efeito benéfico do uso de solução de sulfato de amônio (SA) para pulverização de glyphosate, bem como da solução de U+SA $\left(2,5+7,5 \mathrm{~g} \mathrm{~L}^{-}\right.$ $\left.{ }^{1}\right)$. Estas soluções elevaram o controle obtido pela menor dose do herbicida (540 $\left.\mathrm{g} \mathrm{ha}^{-1}\right)$, igualando-o àquele obtido pela maior dose $\left(1080 \mathrm{~g} \mathrm{ha}^{-1}\right)$.

Tabela 2. Controle percentual de plantas daninhas após aplicação de glyphosate utilizando solução de fertilizantes nitrogenados (uréia - U e sulfato de amônio - SA) como veículos de pulverização. Piracicaba, 2009

\begin{tabular}{|c|c|c|c|c|c|}
\hline \multirow{3}{*}{ Tratamentos } & \multicolumn{2}{|c|}{ Doses } & \multicolumn{3}{|c|}{ Plantas Daninhas ${ }^{(1)}$} \\
\hline & \multirow{2}{*}{$\frac{\text { Herbicida }}{\mathrm{g} \mathrm{ha}^{-1}}$} & \multirow{2}{*}{$\begin{array}{l}\mathrm{FN}^{(2)} \\
\mathrm{g} \mathrm{L}^{-1}\end{array}$} & \multirow{2}{*}{$\begin{array}{c}\text { Comunidade } \\
\text { Mista }\end{array}$} & \multirow{2}{*}{$\begin{array}{l}\text { B. decumbens } \\
\text { BRADC }\end{array}$} & \multirow{2}{*}{$\frac{\text { S. halepense }}{\text { SORHA }}$} \\
\hline & & & & & \\
\hline \multicolumn{6}{|c|}{ Controle percentual aos 14 dias após aplicação } \\
\hline Testemunha sem aplicação & -- & -- & $0,0 \mathrm{C}$ & $0,0 \mathrm{C}$ & $0,0 \mathrm{C}$ \\
\hline Glyphosate $^{3}$ & 540 & -- & $81,6 \mathrm{~B}$ & $32,4 \mathrm{~B}$ & $56,1 \mathrm{~B}$ \\
\hline Glyphosate + SA & 540 & 15,0 & $87,7 \mathrm{~A}$ & $33,0 \mathrm{~B}$ & $82,8 \mathrm{~A}$ \\
\hline Glyphosate + Uréia $(\mathrm{U})$ & 540 & 5,0 & $82,7 \mathrm{~B}$ & $31,2 \mathrm{~B}$ & $59,3 \mathrm{~B}$ \\
\hline Glyphosate + U + SA & 540 & $2,5+7,5$ & $89,0 \mathrm{~A}$ & $33,4 \mathrm{~B}$ & $80,8 \mathrm{~A}$ \\
\hline Glyphosate & 1080 & -- & $92,7 \mathrm{~A}$ & $41,8 \mathrm{~A}$ & $84,8 \mathrm{~A}$ \\
\hline \multirow{2}{*}{\multicolumn{3}{|c|}{$\begin{array}{c}\mathrm{F}_{\text {(trat) }} \\
\mathrm{CV}(\%)\end{array}$}} & $437,77 * *$ & $111,02 * *$ & $105,06^{* *}$ \\
\hline & & & 5,77 & 11,76 & 12,69 \\
\hline \multicolumn{6}{|c|}{ Controle percentual aos 21 dias após aplicação } \\
\hline Testemunha sem aplicação & -- & -- & $0,0 \mathrm{C}$ & $0,0 \mathrm{C}$ & $0,0 \mathrm{C}$ \\
\hline Glyphosate $^{3}$ & 540 & -- & $88,1 \mathrm{~B}$ & $32,3 \mathrm{~B}$ & $66,2 \mathrm{~B}$ \\
\hline Glyphosate + SA & 540 & 15,0 & $94,7 \mathrm{~A}$ & $35,3 \mathrm{~B}$ & $88,0 \mathrm{~A}$ \\
\hline Glyphosate + Uréia (U) & 540 & 5,0 & $89,5 \mathrm{~B}$ & $33,6 \mathrm{~B}$ & $67,1 \mathrm{~B}$ \\
\hline Glyphosate + U + SA & 540 & $2,5+7,5$ & $95,4 \mathrm{~A}$ & $34,6 \mathrm{~B}$ & $84,8 \mathrm{~A}$ \\
\hline Glyphosate & 1080 & - & $95,3 \mathrm{~A}$ & $44,9 \mathrm{~A}$ & $88,7 \mathrm{~A}$ \\
\hline \multirow{2}{*}{\multicolumn{3}{|c|}{$\begin{array}{l}\mathrm{F}(\text { trat) } \\
\mathrm{CV}(\%)\end{array}$}} & $1102,23 * *$ & $97,56 * *$ & $178,69 * *$ \\
\hline & & & 3,63 & 12,70 & 9,41 \\
\hline \multicolumn{6}{|c|}{ Controle percentual aos 28 dias após aplicação } \\
\hline Testemunha sem aplicação & -- & -- & $0,0 \mathrm{C}$ & $0,0 \mathrm{C}$ & $0,0 \mathrm{C}$ \\
\hline Glyphosate $^{(3)}$ & 540 & -- & $89,7 \mathrm{~B}$ & $41,0 \mathrm{~B}$ & $65,7 \mathrm{~B}$ \\
\hline Glyphosate + SA & 540 & 15,0 & $96,2 \mathrm{~A}$ & $46,2 \mathrm{~B}$ & $85,3 \mathrm{~A}$ \\
\hline Glyphosate + Uréia (U) & 540 & 5,0 & $90,1 \mathrm{~B}$ & $39,2 \mathrm{~B}$ & $67,3 \mathrm{~B}$ \\
\hline Glyphosate + U + SA & 540 & $2,5+7,5$ & $96,0 \mathrm{~A}$ & $43,0 \mathrm{~B}$ & $85,5 \mathrm{~A}$ \\
\hline Glyphosate & 1080 & - & $95,3 \mathrm{~A}$ & $56,1 \mathrm{~A}$ & $90,3 \mathrm{~A}$ \\
\hline \multirow{2}{*}{\multicolumn{3}{|c|}{$\begin{array}{c}\mathrm{F}_{\text {(trat) }} \\
\mathrm{CV}(\%)\end{array}$}} & $1949,61 * *$ & $115,69 * *$ & $131,13 * *$ \\
\hline & & & 2,73 & 11,73 & 11,00 \\
\hline
\end{tabular}

${ }^{(1)}$ Médias seguidas por letras iguais, nas colunas, não diferem entre si segundo teste de agrupamento de médias de Scott-Knott, a 5\% de probabilidade; ${ }^{(2)}$ Fertilizantes nitrogenados, em calda de $300 \mathrm{~L} \mathrm{ha}^{-1} ;{ }^{(3)}$ Roundup Original ${ }^{\circledR}, 360$ $\mathrm{g} \mathrm{L}^{-1}$; **significativo a $1 \%$ de probabilidade pelo teste $\mathrm{F}$. 
Carvalho et al.

Na Área 1, as notas de controle foram atribuídas no âmbito geral da comunidade infestante. Contudo vale ressaltar que, visualmente, a espécie Ipomoea nil foi a melhor indicadora da diferença entre os tratamentos. É provável que a maior diferença visual observada para esta espécie seja consequência de sua maior tolerância ao glyphosate, quando comparada a A. tenella, $P$. hysterophorus ou $A$. viridis (Rodrigues \& Almeida, 2005; Lorenzi, 2006).

De acordo com a legislação brasileira e Frans et al. (1986), para que um tratamento herbicida seja considerado adequado em campo, controles superiores a $80 \%$ devem ser alcançados. Neste caso, nenhum tratamento herbicida controlou de forma satisfatória a infestação de $B$. decumbens (Área 2), uma vez que as notas máximas alcançadas foram de $56 \%$, aos 28 DAA, para aplicação de 1080 g e.a. ha ${ }^{-1}$ de glyphosate (Tabela 2). Estes resultados são explicados por Rodrigues \& Almeida (2005) que indicam doses de até 1440 g e.a. ha ${ }^{-1}$ para controle de B. decumbens. Timossi et al. (2006) alcançaram adequado controle de $B$. decumbens, com notas superiores a 90\%, após aplicação de doses de glyphosate iguais ou superiores a 1440 g e.a. $\mathrm{ha}^{-1}$. Na Área 2, não foram observados efeitos benéficos da pulverização de glyphosate com soluções de fertilizantes nitrogenados (Tabela 2).

Com relação ao controle de $S$. halepense, a escolha da dose do herbicida teve importância fundamental para assegurar a completa dessecação da espécie (Tabela 2). A aplicação da menor dose de glyphosate via solução de uréia ou somente com água não foi suficiente para a adequada dessecação de $S$. halepense, com controle máximo da ordem de $67 \%$. Neste caso, a menor dose de glyphosate só proporcionou controles considerados satisfatórios (> 80\%), quando pulverizado com solução de SA ou U+SA, com igualdade ao controle obtido pela maior dose. Indicações de melhor controle de $S$. halepense com aplicação de glyphosate combinado a fertilizantes nitrogenados também foram observadas por Carvalho et al. (2009a).

Salisbury et al. (1991) também avaliaram a eficácia de glyphosate sobre $S$. halepense quando aplicado em mistura com sulfato de amônio. Neste trabalho, o controle obtido pela menor dose de glyphosate (420 g $\left.\mathrm{ha}^{-1}\right)$ foi igualado ao da maior dose $\left(840 \mathrm{~g} \mathrm{ha}^{-1}\right)$ quando o herbicida foi pulverizado combinado a $3,33 \mathrm{~kg} \mathrm{ha}^{-1}$ do fertilizante e somente foi observado antagonismo para aplicação de glyphosate com $9,7 \mathrm{~kg} \mathrm{ha}^{-1}$ de sulfato de amônio. Por outro lado, Hall et al. (2000) não observaram incremento no controle de $S$. halepense decorrente da aplicação de glyphosate com SA, contudo, neste trabalho, foram utilizadas doses reduzidas do fertilizante, de apenas $2,5 \mathrm{~g} \mathrm{~L}^{-1}$, ou seja, cerca de $0,5 \mathrm{~kg} \mathrm{ha}^{-}$ 1 .

São comuns relatos sobre controle diferencial de espécies de plantas daninhas obtidos pelo glyphosate combinado a fertilizantes nitrogenados. Donald (1988) elevou a eficácia e a consistência dos resultados obtidos pelo glyphosate para controle de Hordeum jubatum, quando aplicado com 2,8 $\mathrm{kg} \mathrm{ha}^{-1}$ de SA. Jordan et al. (1997) relataram que a adição da mesma dose de SA à calda de glyphosate melhorou o controle de Sida spinosa e Ipomoea hederacea, contudo não teve efeito sobre Senna obtusifolia, Sesbania exaltata e Echinochloa crus-galli. Pratt et al. (2003) elevaram o controle de Abutilon theophrasti quando glyphosate foi pulverizado combinado ao SA $\left(20 \mathrm{~g} \mathrm{~L}^{-1}\right)$. Neste sentido, Young et al. (2003) e Nurse et al. (2008) também observaram melhor controle de $A$. theophrasti após adição de $20 \mathrm{~g}$ $\mathrm{L}^{-1}$ de $\mathrm{SA}$ à calda do glyphosate, contudo mínima resposta foi observada para Amaranthus retroflexus e Chenopodium album.

A análise da massa fresca residual está em concordância com os dados de controle 
Carvalho et al.

(Tabela 3). Neste caso, para a comunidade mista de plantas daninhas (Área 1), todos os tratamentos de glyphosate que receberam adição de fertilizantes nitrogenados foram diferentes da aplicação da menor dose do produto somente com água. Não foram observadas diferenças entre os tratamentos herbicidas aplicados sobre $B$. decumbens. Para $S$. halepense, melhores resultados foram obtidos com a maior dose de glyphosate (1080 $\mathrm{g} \mathrm{ha}{ }^{-1}$ ), sem diferença para a menor dose aplicada com solução de SA ou de U+SA.
Quanto à análise de massa seca, somente foram observadas vantagens da aplicação de glyphosate com fertilizantes nitrogenados sobre S. halepense (Tabela 3). A massa de plantas daninhas é uma variável com menor sensibilidade a pequenas variações experimentais, porém a adoção de maior número de repetições contribuiu para a ocorrência de diferenças neste trabalho, principalmente quando à análise da massa fresca.

Tabela 3. Massa fresca e seca residual $\left(\mathrm{g} \mathrm{m}^{-2}\right)$ de plantas daninhas após aplicação de glyphosate utilizando solução de fertilizantes nitrogenados (uréia - U e sulfato de amônio - SA) como veículos de pulverização. Piracicaba, 2009

\begin{tabular}{|c|c|c|c|c|c|}
\hline \multirow{3}{*}{ Tratamentos } & \multicolumn{2}{|c|}{ Doses } & \multicolumn{3}{|c|}{ Plantas Daninhas ${ }^{(1)}$} \\
\hline & Herbicida & $\mathrm{FN}^{(2)}$ & \multirow{2}{*}{$\begin{array}{c}\text { Comunidade } \\
\text { Mista }\end{array}$} & \multirow{2}{*}{$\begin{array}{c}\text { B. decumbens } \\
\text { BRADC }\end{array}$} & \multirow{2}{*}{$\begin{array}{c}\text { S. halepenst } \\
\text { SORHA }\end{array}$} \\
\hline & $\mathrm{g} \mathrm{ha}^{-1}$ & $\mathrm{~g} \mathrm{~L}^{-1}$ & & & \\
\hline \multicolumn{6}{|c|}{ Massa fresca residual aos 28 dias após aplicação $\left(\mathrm{g} \mathrm{m}^{-2}\right)$} \\
\hline $\begin{array}{l}\text { Testemunha sem } \\
\text { aplicação }\end{array}$ & -- & -- & $3012,2 \mathrm{C}$ & $1871,6 \mathrm{~B}$ & $2105,9 \mathrm{C}$ \\
\hline Glyphosate $^{(3)}$ & 540 & -- & $1797,0 \mathrm{~B}$ & $1419,8 \mathrm{~A}$ & 988,6 B \\
\hline Glyphosate + SA & 540 & 15,0 & $1148,0 \mathrm{~A}$ & $1166,8 \mathrm{~A}$ & $530,2 \mathrm{~A}$ \\
\hline Glyphosate + Uréia (U) & 540 & 5,0 & $1438,0 \mathrm{~A}$ & $1325,3 \mathrm{~A}$ & $720,3 \mathrm{~B}$ \\
\hline Glyphosate + U + SA & 540 & $2,5+7,5$ & $1341,6 \mathrm{~A}$ & $1387,6 \mathrm{~A}$ & $557,1 \mathrm{~A}$ \\
\hline Glyphosate & 1080 & -- & $1090,3 \mathrm{~A}$ & $1017,7 \mathrm{~A}$ & $357,3 \mathrm{~A}$ \\
\hline \multicolumn{3}{|c|}{$\mathrm{F}_{\text {(trat) }}$} & $20,09 * *$ & 3,86 ** & $33,51 * *$ \\
\hline \multirow{2}{*}{\multicolumn{3}{|c|}{ eca residual aos $28 \mathrm{~d}$}} & 23,97 & 26,54 & 30,84 \\
\hline & & & \multicolumn{3}{|c|}{ seca residual aos 28 dias após aplicação $\left(\mathrm{g} \mathrm{m}^{-2}\right)$} \\
\hline $\begin{array}{l}\text { Testemunha sem } \\
\text { aplicação }\end{array}$ & -- & -- & $863,03 \mathrm{~B}$ & 639,8 & $569,7 \mathrm{C}$ \\
\hline Glyphosate $^{3}$ & 540 & -- & $513,7 \mathrm{~A}$ & 545,3 & $388,9 \mathrm{~B}$ \\
\hline Glyphosate + SA & 540 & 15,0 & $442,6 \mathrm{~A}$ & 461,4 & $260,2 \mathrm{~A}$ \\
\hline Glyphosate + Uréia (U) & 540 & 5,0 & $520,1 \mathrm{~A}$ & 507,6 & $266,6 \mathrm{~A}$ \\
\hline Glyphosate + U + SA & 540 & $2,5+7,5$ & $457,4 \mathrm{~A}$ & 561,0 & $289,1 \mathrm{~A}$ \\
\hline Glyphosate & 1080 & -- & $397,7 \mathrm{~A}$ & 477,5 & $212,3 \mathrm{~A}$ \\
\hline \multirow{2}{*}{\multicolumn{3}{|c|}{$\begin{array}{c}\mathrm{F}_{\text {(trat) }} \\
\mathrm{CV}(\%)\end{array}$}} & $12,59 * *$ & $1,55^{\mathrm{NS}}$ & $12,11 * *$ \\
\hline & & & 21,82 & 24,05 & 27,77 \\
\hline
\end{tabular}

${ }^{(1)}$ Médias seguidas por letras iguais, nas colunas, não diferem entre si segundo teste de agrupamento de médias de Scott-Knott, a 5\% de probabilidade; ${ }^{(2)}$ Fertilizantes nitrogenados, em calda de $300 \mathrm{~L} \mathrm{ha}^{-1}$; ${ }^{(3)}$ Roundup Original ${ }^{\circledR}, 360$ $\mathrm{g} \mathrm{L}^{-1} ; * *$ significativo a $1 \%$ de probabilidade pelo teste $\mathrm{F} ;{ }^{\text {NS }}$ Não significativo.

A adoção da solução de U+SA como veículo de pulverização de glyphosate teve resultados positivos sobre a comunidade mista
(Área 1) e sobre S. halepense (Área 3), porém nunca superiores à solução somente com SA. Carvalho et al. (2010), por outro lado, 
Carvalho et al.

relataram maior eficácia do herbicida glyphosate sobre Digitaria insularis quando pulverizado com solução de U+SA $(2,5+7,5 \mathrm{~g}$ $\left.\mathrm{L}^{-1}\right)$. Ainda, Suwunnamek \& Parker (1975) observaram que a adição de uréia, fosfatobutil-amônio e fosfato de amônio elevaram o controle de tiririca (Cyperus rotundus) pelo glyphosate, porém sem vantagens em relação ao SA.

Com frequência, espécies daninhas pertencentes ao mesmo gênero de plantas têm resposta diferencial a determinado tratamento herbicida. Por exemplo, estudos demonstraram diferenças de susceptibilidade de espécies do gênero Ipomoea aos herbicidas bentazon (McClelland et al., 1978) e ao carfentrazoneethyl (Christoffoleti et al., 2006); de Bidens a chlorimuron-ethyl e imazathapyr (LópezOvejero et al., 2006); e de espécies de Amaranthus ao trifloxysulfuron-sodium e chlorimuron-ethyl (Carvalho, 2006). A diferença de suscetibilidade de espécies de plantas daninhas a herbicidas aplicados em pós-emergência pode estar relacionada com a afinidade enzimática das moléculas; com a absorção, translocação ou exclusão diferencial dos herbicidas; ou mesmo com rotas de detoxificação metabólica (Carvalho, 2006). Assim, a exposição de diferentes espécies ao mesmo tratamento herbicida tem grande importância prática, pois subsidia estimativa das condições em que cada tratamento será mais ou menos eficaz.

Assim, sugere-se que incrementos na eficácia do glyphosate sobre plantas daninhas poderão ser obtidos com adoção de solução de fertilizantes nitrogenados como veículo de pulverização, mesmo utilizando água com baixa concentração de sais, principalmente devido à adição do SA sobre espécies de difícil controle, tais como $S$. halepense e aquelas pertencentes ao gênero Ipomoea. A exceção foi $B$. decumbens que, embora necessite de doses mais altas de glyphosate, não teve maior controle com uso combinado do herbicida e fertilizantes nitrogenados. Ainda, também vale destacar que não foram observados efeitos antagônicos das diferentes misturas utilizadas. A solução de U+SA tem potencial para uso agrícola visando elevar a eficácia do glyphosate, de modo que novos ajustes nas proporções de U e SA devem ser estudados, tais como $5: 10$ ou $7,5: 7,5 \mathrm{~g} \mathrm{~L}^{-1}$, respectivamente.

\section{Conclusões}

O uso de solução de fertilizantes nitrogenados como veículo para aplicação do glyphosate não elevou o controle de Brachiaria decumbens;

A menor dose do herbicida glyphosate (540 g e.a. ha $^{-1}$ ) teve maior eficácia sobre a comunidade mista de plantas daninhas e Sorghum halepense devido a pulverização com solução de sulfato de amônio $\left(15 \mathrm{~g} \mathrm{~L}^{-1}\right)$ ou de uréia + sulfato de amônio $\left(2,5+7,5 \mathrm{~g} \mathrm{~L}^{-1}\right)$;

Os resultados positivos obtidos pela aplicação de glyphosate com fertilizantes nitrogenados também foram observados para a redução de massa fresca da comunidade mista e para massa fresca e seca de S. halepense.

\section{Agradecimentos}

Ao Conselho Nacional de Desenvolvimento Científico e Tecnológico CNPq, pela concessão de bolsas.

\section{Referências}

ASOCIATION LATINOAMERICANA DE MALEZAS. Recomendaciones sobre unificación de los sistemas de evaluación en ensayos de control de malezas. ALAM, v.1, p. 35-38, 1974

CARVALHO, S.J.P. Características biológicas e suscetibilidade a herbicidas de cinco espécies de plantas daninhas do gênero 
Carvalho et al.

Amaranthus. 2006. 96p. Dissertação (Mestrado em Agronomia) - Escola Superior de Agricultura "Luiz de Queiroz", Universidade de São Paulo, Piracicaba, 2006.

CARVALHO, S.J.P. et al. Glifosato aplicado com diferentes concentrações de uréia ou sulfato de amônio para dessecação de plantas daninhas. Pesquisa Agropecuária Brasileira, v.43, n.11, p.1501-1508, 2008.

CARVALHO, S.J.P. et al. Dessecação de plantas daninhas com glyphosate em mistura com uréia ou sulfato de amônio. Planta Daninha, v.27, n.2, p.353-361, 2009a.

CARVALHO, S.J.P. et al. Eficácia e pH de caldas de glifosato após adição de fertilizantes nitrogenados e utilização de pulverizador pressurizado por $\mathrm{CO}_{2}$. Pesquisa Agropecuária Brasileira, v.44, n.6, p.569-575, 2009 b.

CARVALHO, S.J.P. et al. Adição simultânea de sulfato de amônio e uréia à calda de pulverização do herbicida glyphosate. Planta Daninha, v.28, n.3, p.575-584, 2010.

CHRISTOFFOLETI, P.J. et al. Glyphosate sustainability in South American cropping systems. Pest Management Science, v.64, n.4, p.422-427, 2008.

CHRISTOFFOLETI, P.J. et al. Carfentrazoneethyl aplicado em pós-emergência para o controle de Ipomoea spp. e Commelina benghalensis na cultura da cana-de-açúcar. Planta Daninha, v.24, n.1, p.83-90, 2006.

DONALD, W.W. Established foxtail barley, Hordeum jubatum, control with glyphosate plus ammonium sulfate. Weed Technology, v.2, n.3, p.364-368, 1988.

EMBRAPA - EMPRESA BRASILEIRA DE PESQUISA AGROPECUÁRIA. Centro Nacional de Pesquisa de Solos. Sistema brasileiro de classificação de solos. 2.ed. Rio de Janeiro: EMBRAPA, 2006. 306p.

FRANS, R.E. et al. Experimental design and techniques for measuring and analyzing plant responses to weed control practices. In: CAMPER, N.D. (Ed.) Research methods in weed science. 3.ed. Champaign: Southern Weed Science Society, 1986. p.29-46.

FRANSON, M.A.H. Standard methods for the examination of water and wastewater. 19.ed. Washington: American Public Heath Association, 1995. 1074p.

GAUVRIT, C. Glyphosate response to calcium, ethoxylated amine surfactant, and ammonium sulfate. Weed Technology, v.17, n.4, p.799-804, 2003.

GEIGER, D. R.; FUCHS, M. A. Inhibitors of aromatic amino acid biosynthesis (glyphosate). In: BÖGER, P.; WAKABAYASHI, K.; HIRAI, K. (Eds.) Herbicide classes in development. Berlin: Springer-Verlag, 2002. p. 59-85.

GRONWALD, J.W. et al. Effect of ammonium sulfate on absorption of imazethapyr by quackgrass (Elytrigia repens) and maize (Zea mays) cell suspension cultures. Weed Science, v.41, n.3, p.325-334, 1993.

HALL, G.J.; HART, C.A.; JONES, C.A. Plants as sources of cations antagonistic to glyphosate activity. Pest Management Science, London, v.56, n.4, p.351-358, 2000.

JORDAN, D.L. et al. Influence of application variables on efficacy of glyphosate. Weed Technology, v.11, n.2, p.354-362, 1997.

LÓPEZ-OVEJERO, R.F. et al. Resistance and differential susceptibility of Bidens pilosa and B. subalternans biotypes to ALS-inhibiting herbicides. Scientia Agricola, v. 63, n. 2, p. 139-145, 2006.

LORENZI, H. Manual de identificação e controle de plantas daninhas: plantio direto e convencional. 6.ed. Nova Odessa: Instituto Plantarum, 2006. 339 p.

MacISAAC, S.A.; PAUL, R.N.; DEVINE, M.D. A scanning electron microscope study of 
Carvalho et al.

glyphosate deposits in relation to foliar uptake. Pesticide Science, v.31, n.1, p.53-64, 1991.

McCLELLAND, M.R. et al. Responses of six morningglory (Ipomoea) species to bentazon. Weed Science, v. 26, n. 5, p. 459-464, 1978.

MONQUERO, P.A. et al. Absorção, translocação e metabolismo do glyphosate por plantas tolerantes e suscetíveis a este herbicida. Planta Daninha, v.22, n.3, p.445-451, 2004.

NALEWAJA, J.D.; MATYSIAK, R. Salt antagonism of glyphosate. Weed Science, v.39, n.4, p.622-628, 1991.

NALEWAJA, J.D.; MATYSIAK, R.; FREEMAN, T.P. Spray droplet residual of glyphosate in various carriers. Weed Science, v.40, n.4, p.576-589, 1992.

NURSE, R.E. et al. Annual weed control may be improved when AMS is added to belowlabel glyphosate doses in glyphosate-tolerant maize (Zea mays L.). Crop Protection, v.27, n.3-5, p.452-458, 2008.

O'SULLIVAN， P.A.; O'DONOVAN， J.T.; HAMMAN, W.M. Influence of non-ionic surfactants, ammonium sulphate, water quality and spray volume on the phytotoxicity of glyphosate. Canadian Journal of Plant Science, v.61, n.2, p.391-400, 1981.

PRATT, D.; KELLS, J.J.; PENNER, D. Substitutes for ammonium sulfate as additives with glyphosate and glufosinate. Weed Technology, v.17, n.3, p.576-581, 2003.

RAMSDALE, B.K.; MESSERSMITH, C.G.; NALEWAJA, J.D. Spray volume, formulation, ammonium sulfate, and nozzle effects on glyphosate efficacy. Weed Technology, v.17, n.3, p.589-598, 2003.

RODRIGUES, B.N.; ALMEIDA, F.S. Guia de herbicidas. 5.ed. Londrina, 2005. 592p.

RUITER, H.D.; MEINEN, E. Adjuvantincreased glyphosate uptake by protoplasts isolated from quackgrass Elytrigia repens (L.)
Nevski. Weed Science, v.44, n.1, p.38-45, 1996.

SALAS-PINO, P.A.G. Efeito de adjuvantes, volume de calda e qualidade da água na eficácia do herbicida glyphosate. 1996. 79p. Dissertação (Mestrado em Agronomia) Escola Superior de Agricultura "Luiz de Queiroz”, Universidade de São Paulo, Piracicaba, 1996.

SALISBURY， C.D.; CHANDLER， J.M.; MERKLE, M.G. Ammonium sulfate enhancement of glyphosate and SC-0224 control of johnsongrass (Sorghum halepense). Weed Technology, v.5, n.1, p.18-21, 1991.

SCOTT, A.J.; KNOTT, M.A. Cluster analysis method for grouping means in the analysis of variance. Biometrics, v.30, n.2, p.507-512, 1974.

STORCK, L. et al. Experimentação vegetal. Santa Maria: Ed. UFSM, 2006. 198p.

SUWUNNAMEK, U.; PARKER, C. Control of Cyperus rotundus with glyphosate: the influence of ammonium sulfate and other additives. Weed Research, v.15, n.1, p.13-20, 1975.

THELEN, K.D.; JACKSON, E.P.; PENNER, D. The basis for the hard-water antagonism of glyphosate activity. Weed Science, v.43, n.4, p.541-548, 1995.

TIMOSSI, P.C.; DURIGAN, J.C.; LEITE, G.J. Eficácia de glyphosate em plantas de cobertura. Planta Daninha, v.24, n.3, p.475-480, 2006.

WITTE, C.P. et al. Leaf urea metabolism in potato. Urease activity profile and patterns of recovery and distribution of ${ }^{15} \mathrm{~N}$ after foliar urea application in wild-type and ureaseantisense transgenics. Plant Physiology, v.128, n.3, p.1129-1136, 2002.

YAMADA, Y.; WITTWER, S.H.; BUKOVAC, M.J. Penetration of organic compounds through isolated cuticular 
Carvalho et al.

membranes with special reference to $\mathrm{C}^{14}$ urea.

Plant Physiology, v.40, n.1, p.170-175, 1965.

YOUNG, B.G. et al. Glyphosate translocation

in common lambsquarters (Chenopodium album) and velvetleaf (Abutilon theophrasti) in response to ammonium sulfate. Weed Science, v.51, n.2, p.151-156, 2003. 